\title{
Parotid lymphoepithelial cyst in non-HIV patient.
}

\author{
Carlos Augusto Ferreira Alves ${ }^{1}$, Ophir Ribeiro Júnior ${ }^{2}$, Alexandre Meireles Borba ${ }^{2}$, Suzana Cantanhede \\ Orsini Machado de Souza ${ }^{3}$, Maria da Graça Naclério-Homem ${ }^{4}$
}

${ }^{1}$ DDS, MSc. Staff in Oral \& Maxillofacial Surgery - Hospital Universitário da Universidade de São Paulo - USP

${ }^{2}$ DDS, MSc. PhD student in Oral and Maxillofacial Surgery - Faculdade de Odontologia da Universidade de São Paulo - USP

${ }^{3}$ DDS, PhD. Head Professor in Oral Pathology - Faculdade de Odontologia da Universidade de São Paulo - USP

${ }^{4}$ DDS, PhD. Associate Professor in Oral and Maxillofacial Surgery - Faculdade de Odontologia da Universidade de São Paulo USP

Correspondence:

Rua Los Angeles, 431, Jardim Califórnia

Cuiabá - MT, Brazil. CEP 78070-400

E-mail address: alex80@terra.com.br

\author{
Alves CAF, Ribeiro Júnior O, Borba AM, Souza SCOM, Naclério-Homem \\ MG. Parotid lymphoepithelial cyst in non-HIV patient. J Clin Exp J Clin \\ Exp Dent. 2011;3(Suppl1):e400-3. \\ http://www.medicinaoral.com/odo/volumenes/v3iSuppl1/jcedv3iSu- \\ ppl1p400.pdf
}

\begin{abstract}
The lymphoepithelial cyst is a dysontogenic benign cyst originated from the epithelial remnant retained inside the lymphoid tissue during the embryogenesis that occurs in the lateral neck region and, less frequently, in the oral cavity and in the parotid gland. The parotid gland evolvement increased significantly after the HIV discovery and it is related to ductal obstruction phenomenon that goes along with a follicular hyperplasia in the periductal parotid lymph nodes. However, lymphoepithelial cysts are rare in non-HIV patients. Treatment options are controversial and might include cystic content aspiration, sclerosis-therapy, radiotherapy and surgery. Enucleation of the cyst while preserving the gland might not be resolutive in all cases because the cyst tends to reoccur in other areas of the parotid gland but recurrence appears to be related to tissue alterations promoted by the HIV, therefore not applicable to non-HIV lesions. This article presents a case of parotid lymphoepithelial cyst in a non-HIV patient, highlighting the importance of serum investigation for diagnosis purpose.
\end{abstract}

Key words: Salivary gland; Parotid Gland; Lymphoepithelial Cyst; Oral Pathology; HIV. 


\section{Introduction}

The lymphoepithelial cyst (LEC) is a dysontogenic benign cyst originated from the epithelial remnant retained in lymphoid tissues during the embryogenesis, although it might also be related to branchial cleft epithelium $(1,2)$. It presents a predilection to the lateral neck region, occurring less frequently in the oral cavity or in the parotid gland. These parotid cysts are usually seen in HIVpositive patients, generally as one of the signs of this infection (3-5).

The occurrence of LEC corresponded to less than 3\% of parotid gland benign lesions in the 70's but it presented a significantly incidence increase after the discovery of the HIV (6). Due to the rarity of the LEC in non-HIV patients, it is proposed here a report of such case.

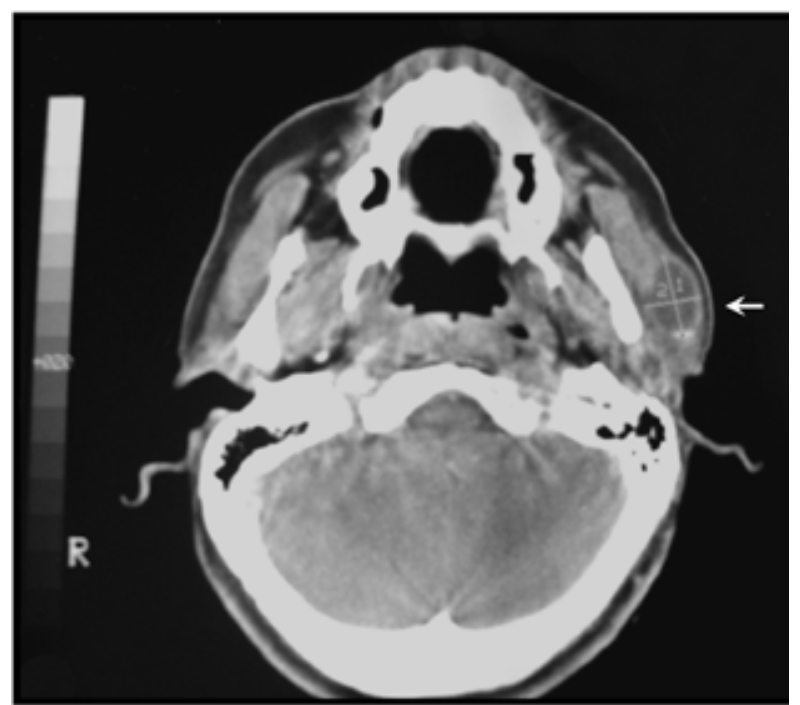

Fig. 1. Tomographic aspect, in axial view, displaying a cyst-like image at the left parotid gland.

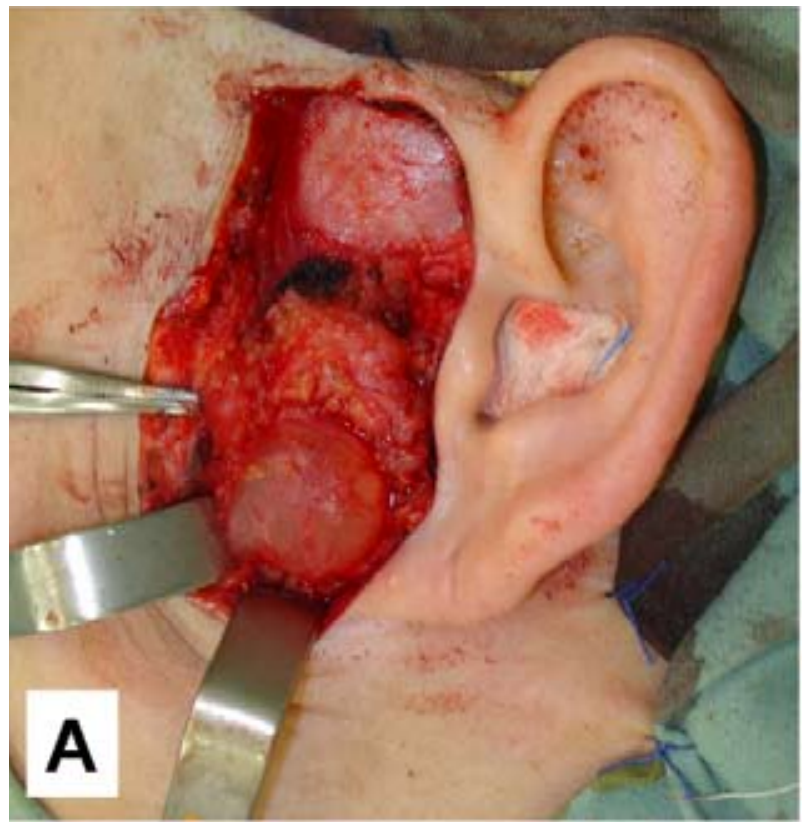

\section{Case Report}

A middle-aged white woman presented for evaluation of an odontogenic abscess. During clinical examination, in spite of the abscess, a firm, movable, not painful $3 \mathrm{~cm}$ nodule was observed in the parotid-masseteric left region. No function impairment of the facial nerve was seen.

After the treatment of the infection, the parotid nodule was investigated for possible neoplasm, cyst or salivary retention phenomenon. The $\mathrm{CT}$ scan demonstrated a circular image at the superficial lobe of the parotid gland with lower density than the gland tissue, indicating the cyst-like aspect (Fig. 1).

The enucleation of the lesion was accomplished through a pre-auricular incision, which confirmed the cyst-like aspect, intimately related to the parotid duct (Fig. 2). Histopathological examination demonstrated a cyst covered by stratified epithelium with multiple germinative lymphoid centers in the conjunctive capsule, thus given the diagnosis of LEC (Fig. 3). HIV antibodies were investigated by the ELISA test with a negative result. After three years, no recurrence nor the formation of other new cyst were observed.

\section{Discussion}

It is considered that the epithelial remnant of the parotid gland can originate LEC inside the parotid gland and cervical lymph nodes whereas the epithelial retention in oropharyngeal lymphoid organs - normal or ectopic - justifies the occurrence of intraoral cysts (7-9). Such hypothesis was previously demonstrated with the experimental development of LECs in hamsters after oral epithelium implantation inside cervical lymph nodes (10).

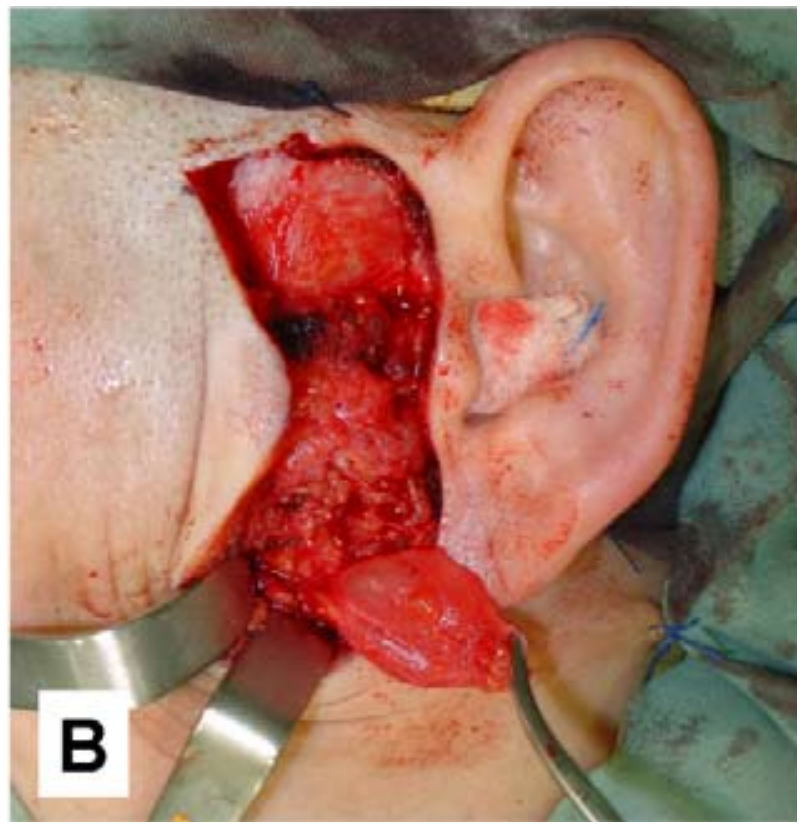

Fig. 2. Transoperatory view demonstrating the cyst-like aspect of the parotid nodule (A) and its surgical removal (B). 

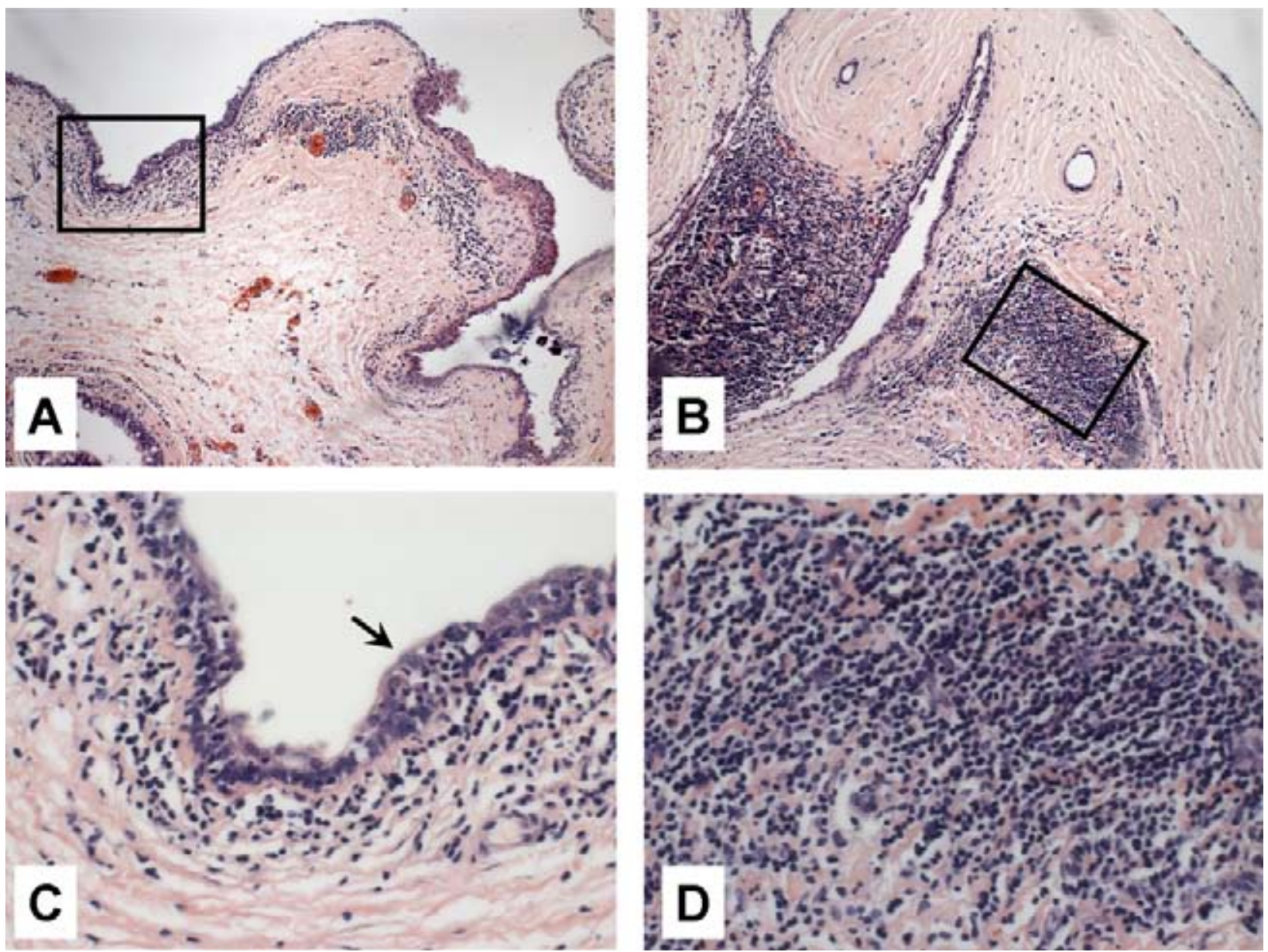

Fig. 3. Histopathological aspect, in hematoxylin-eosin stain, showing a cystic lesion covered with stratified epithelium (A), lymphoid germinal centers in the connective tissue capsule (B); 10x magnification. Images $C$ and D display the epithelium (arrow) and the lymphoid germinal centers (as seen in previous highlighted areas in $A$ and B, respectively); 40x magnification.

Ductal obstruction phenomena are referred to the pathogenesis of parotid LEC associated with the HIV. This obstruction goes along with a follicular hyperplasia in the periductal parotid lymph nodes, induced by the virus, which reminds the persistent lymphopathy observed in patients manifesting AIDS (4). Curiously, a close anatomic relationship with the parotid duct was surgically observed in the present case, even though the patient was negative for HIV test.

Parotid LECs are characterized by a non-painful loose augmentation in the affected gland (3). There are two peculiarities that distinguish the cyst associated with HIV: frequent bilateral occurrence and multicystic appearance observed in CT or MRI (6). The greatest problem related to the LEC in HIV positive patients is the progression to a lymphoma (3), which increases the responsibility for HIV infection investigation, as in the presented case.

For parotid LECs associated with HIV, treatment options are controversial and might include cyst content aspiration, sclerosis-therapy, radiotherapy and surgery $(3,6,11,12)$. Aspiration is a palliative treatment modality aiming aesthetic results, although the cyst recovers their volume after a while. The importance of this technique is the possibility of cytological examination, thus helping the diagnosis with minimum distress for the patient $(3,12)$. Intralesional injections of doxycycline promote sclerosis and appear to be effective in the regression of some unicystic lesions, the reason why it has been used in the pediatric population (12). Radiotherapy is controversial due to the possible malign differentiation of benign lesions (6) so a lesion not associated with HIV infection might not worth the risk of such collateral damage. Enucleation of the cyst while preserving the gland might not be resolutive because the cyst tends to reoccur in other areas of the parotid gland $(6,12)$. However, recurrence appears to be related to tissue alterations promoted by the HIV, therefore not applicable to non-HIV lesions. Other surgical treatment is the superficial removal of the parotid gland with preservation of the facial nerve, which can be a challenging procedure $(6,12)$.

It is reasonable to consider that is better to remove an asymptomatic lesion before any secondary infection interferes with the surgical procedure (11). Parotid LECs not related with HIV present better prognosis with pro- 
bable resolution after enucleation since there is no other underlying cause. The presented technique seems to be effective with low risks of recurrence; however HIV test must be used to exclude the viral infection.

\section{References}

1. Apel RL, Asa SL, Chalvardjian A, LiVolsi VA. Intrathyroidal lymphoepithelial cysts of probable branchial origin. Hum Pathol. 1994;25:1238-42.

2. Glosser JW, Pires CAS, Feinberg SE. Branchial cleft or cervical lymphoepithelial cysts: etiology and management. J Am Dent Assoc. 2003;134:81-6.

3. Mandel L, Hong J. HIV-associated parotid lymphoepithelial cysts. J Am Dent Assoc. 1999;130:528-32.

4. Vargas PA, Villalba H, Passos AP, Saldiva PH, Mauad T, Caiaffa Filho HH, et al. Simultaneous occurrence of lymphoepithelial cysts, cytomegalovirus and mycobacterial infections in the intraparotid lymph nodes of a patient with AIDS. J Oral Pathol Med. 2001;30:507-9.

5. Favia G, Capodiferro S, Scivetti M, Lacaita MG, Filosa A, Lo Muzio L. Multiple parotid lymphoepithelial cysts in patients with HIV-infection: report of two cases. Oral Dis. 2004;10:151-4.

6. Kooper DP, Leemans CR, Hulshof MC, Claessen FA, Snow GB. Management of benign lymphoepithelial lesions of the parotid gland in human immunodeficiency virus-positive patients. Eur Arch Otohinolaryngol. 1998;255:427-9.

7. Buchner A, Hansen LS. lymphoepithelial cysts of the oral cavity: a clinicopathologic study of thirty-eight cases. Oral Surg Oral Med Oral Pathol. 1980;50:441-9.

8. Sakoda S, Kodama Y, Shiba R. lymphoepithelial cyst of oral cavity: report of a case and review of the literature. Int J Oral Surg. 1983;12:127-31.

9. Camilleri AC, Lloyd RE. Lymphoepithelial cyst of the parotid gland. Br J Oral Maxillofac Surg. 1990;28:329-32.

10. Vickers RA, von der Muhll O. An investigation concerning inducibility of lymphoepithelial cyst in hamsters by autogenous epithelial transplantation. J Dent Res. 1966;45:1029-32.

11. Glosser JW, Pires CA, Feinberg SE. Branchial cleft or cervical lymphoepithelial cysts: etiology and management. J Am Dent Assoc. 2003;134:81-6.

12. Kumar VV, Sharma N. Parotid lymphoepithelial cysts as an indicator of HIV infection. J Can Dent Assoc. 2011;77:b28. 